\title{
On the mean value problem for linear functional differential equations in Banach spaces
}

\author{
Andrei Ronto
}




\title{
ON THE MEAN VALUE PROBLEM FOR LINEAR FUNCTIONAL DIFFERENTIAL EQUATIONS IN BANACH SPACES
}

\author{
ANDREi RonTo \\ Institute of Mathematics, Ukrainian Academy of Sciences \\ 3 Tereshchenkovskaya St., 01601 Kiev, Ukraine \\ ar@imath.kiev.ua
}

[Received: December 26, 2001]

\begin{abstract}
We establish some unique solvability results concerning the mean value problem for a linear functional differential equation in a Banach space.
\end{abstract}

Mathematical Subject Classification: 34K06, 34K07, 34K10

Keywords: linear functional differential equation, Lyapunov-Schmidt method, spectral radius, mean value problem

\section{Problem description}

We consider the boundary value problem

$$
\begin{gathered}
u^{\prime}(t)=(l u)(t)+q(t), \quad t \in[a, b], \\
\frac{1}{b-a} \int_{a}^{b} u(\sigma) \mathrm{d} \sigma=u(\tau)+\eta,
\end{gathered}
$$

where $q \in L([a, b], X)$ and $\eta \in X$. Here and below, $\tau$ is a certain (fixed) point from $[a, b],\langle X,\|\cdot\|\rangle$ is a Banach space, and $l: C([a, b], X) \rightarrow L([a, b], X)$ is a linear operator satisfying the following

Assumption 1. There exists a function $\beta \in L([a, b], \mathbb{R})$ such that

$$
\|(l u)(t)\| \leq \beta(t) \max _{\sigma \in[a, b]}\|u(\sigma)\|
$$

for all $u \in C([a, b], X)$ and a.e. $t \in[a, b]$.

Remark 2. It follows immediately from (1.3) that, under Assumption 1, the operator $l$ is continuous.

By a solution of (1.1), (1.2), we mean an absolutely continuous function $x:[a, b] \rightarrow$ $X$ possessing property (1.2) and satisfying (1.1) almost everywhere on $[a, b]$. It is natural to refer to system (1.1), (1.2) as to the linear inhomogeneous mean value problem. 
Along with (1.1), (1.2), we shall also consider the corresponding homogeneous mean value problem

$$
\begin{gathered}
u^{\prime}(t)=(l u)(t), \quad t \in[a, b], \\
\frac{1}{b-a} \int_{a}^{b} u(\sigma) \mathrm{d} \sigma=u(\tau) .
\end{gathered}
$$

\section{Notation}

Let $\langle X,\|\cdot\|\rangle$ be a Banach space. The following basic notation is used throughout the paper.

(1) $\mathcal{B}(X)$ is the algebra of all linear bounded operators on $X$.

(2) $1_{X}$ is the unity in $\mathcal{B}(X)$.

(3) $\operatorname{ker} A($ resp., $\operatorname{im} A)$ is the kernel (resp., image) of $A \in \mathcal{B}(X)$.

(4) $r(A)$ is the spectral radius of $A \in \mathcal{B}(X)$.

(5) $C([a, b], X)$ is the Banach space of all the continuous mappings from $[a, b]$ to $X$ with the norm

$$
C([a, b], X) \ni u \longmapsto \max _{t \in[a, b]}\|u(t)\| ;
$$

(6) $L([a, b], X)$ is the Banach space of all the Bochner integrable mappings from $[a, b]$ to $X$ with the norm

$$
L([a, b], X) \ni u \longmapsto \int_{a}^{b}\|u(t)\| \mathrm{d} t .
$$

\section{Definitions, assumptions, and subsidiary results}

Let us introduce a definition.

Definition 3. Given a linear operator $l: C([a, b], X) \rightarrow L([a, b], X)$, we define the function $l^{\square}:[a, b] \rightarrow \mathcal{B}(X)$ by putting

$$
l^{\square}=l 1_{X} .
$$

Remark 4. In other words, the action of the restriction of $l$ to the subspace of constant functions is nothing but the multiplication by $l^{\square}$.

From now on, we make the following

Assumption 5. The linear operator $\Lambda_{l, \tau}: X \rightarrow X$ defined by the formula

$$
\Lambda_{l, \tau}:=\int_{a}^{b}\left(\int_{\tau}^{t} l^{\square}(\sigma) \mathrm{d} \sigma\right) \mathrm{d} t
$$

is invertible. 
The invertibility of operator (3.2) allows us to put

$$
\begin{aligned}
{\left[\Gamma_{l, \tau} y\right](t):=\int_{\tau}^{t} y(\sigma) \mathrm{d} \sigma } & \\
& -\int_{\tau}^{t} l^{\square}(\sigma) \mathrm{d} \sigma \Lambda_{l, \tau}^{-1} \int_{a}^{b}\left(\int_{\tau}^{s} y(\sigma) \mathrm{d} \sigma\right) \mathrm{d} s
\end{aligned}
$$

for $y \in L([a, b], X)$ and $t \in[a, b]$. [The integral in (3.2) is understood in the Bochner sense, whereas $l^{\square}$ is related to $l$ according to formula (3.1).]

Consequently, it is easy to see that formula (3.3) determines a linear, continuous operator $\Gamma_{l, \tau}$ from $L([a, b], X)$ to $C([a, b], X)$.

Lemma 6. The inclusion

$$
X \subset \operatorname{ker} \Gamma_{l, \tau} l
$$

holds.

Remark 7. Relation (3.4) from Lemma 6 should be understood in the sense that the constant mappings $[a, b] \rightarrow X$ are identified with the corresponding vectors from $X$.

Proof of Lemma 6. According to Definition 3, for an arbitrary $x \in X$, we have

$$
(l x)(t)=l^{\square}(t) x, \quad t \in[a, b] .
$$

Therefore, for all $x \in X$, formula (3.3) yields

$$
\begin{aligned}
\Gamma_{l, \tau} l x & =\int_{\tau}^{t}(l x)(\sigma) \mathrm{d} \sigma-\int_{\tau}^{t} l^{\square}(\sigma) \mathrm{d} \sigma \Lambda_{l, \tau}^{-1} \int_{a}^{b}\left(\int_{\tau}^{s}(l x)(\sigma) \mathrm{d} \sigma\right) \mathrm{d} s \\
& =\int_{\tau}^{t} l^{\square}(\sigma) x \mathrm{~d} \sigma-\int_{\tau}^{t} l^{\square}(\sigma) \mathrm{d} \sigma \Lambda_{l, \tau}^{-1} \int_{a}^{b}\left(\int_{\tau}^{s} l^{\square}(\sigma) x \mathrm{~d} \sigma\right) \mathrm{d} s \\
& =\left[\int_{\tau}^{t} l^{\square}(\sigma) \mathrm{d} \sigma-\int_{\tau}^{t} l^{\square}(\sigma) \mathrm{d} \sigma \Lambda_{l, \tau}^{-1} \int_{a}^{b}\left(\int_{\tau}^{s} l^{\square}(\sigma) \mathrm{d} \sigma\right) \mathrm{d} s\right] x,
\end{aligned}
$$

which, combined with (3.2), implies that $\Gamma_{l, \tau} l x=0$.

Remark 8. Lemma 6 claims that $\left.\operatorname{im} l\right|_{X} \subset \operatorname{ker} \Gamma_{l, \tau}$.

Lemma 9. For an arbitrary y from $L([a, b], X)$, the equality

$$
\left(\Gamma_{l, \tau} u\right)(\tau)=0
$$

holds.

Proof. Relation (3.5) is an immediate consequence of formula (3.3).

Let $T_{f}$ denote the "shift" mapping

$$
u \longmapsto T_{f} u:=u+f,
$$

where $f$ is a fixed element of $L([a, b], X)$. 
Lemma 10. Let us assume that $l: C([a, b], X) \rightarrow L([a, b], X)$ and $A: L([a, b], X) \rightarrow$ $C([a, b], X)$ are certain linear operators satisying the condition ${ }^{1}$

$$
X \subset \operatorname{ker} A l .
$$

Then, for every $N \geq 1$,

$$
\left(T_{\xi} A l\right)^{N}=T_{\xi}(A l)^{N}
$$

where $\xi \in X$ is arbitrary and the mapping $T_{\xi}: C([a, b], X) \rightarrow C([a, b], X)$ is defined according to relation (3.6).

Proof. Let us fix arbitrary $\xi \in X$ and $u_{0} \in C([a, b], X)$, and set

$$
u_{N}:=\left(T_{\xi} A l\right)^{N} u_{0}, \quad N=0,1,2, \ldots
$$

In view of definition (3.6) and condition (3.7), we have

$$
u_{1}=\xi+A l u_{0}
$$

and

$$
u_{2}=\xi+A l\left[\xi+A l u_{0}\right]=\xi+A\left[l \xi+l A l u_{0}\right]=\xi+(A l)^{2} u_{0} .
$$

Arguing similarly, we can show that

$$
u_{N}=\xi+(A l)^{N} u_{0}
$$

for all $u_{0} \in C([a, b], X), \xi \in X$, and $N \in \mathbb{N}$. This means that equality (3.8) is true.

\section{Reduction of the homogeneous problem (1.4), (1.5) to a family of fixed-point type equations}

Lemma 11. Let $l$ in (1.1) be such that the corresponding operator (3.2) is invertible. If $u \in C([a, b], X)$ is a solution of the mean value problem (1.4), (1.5), then the equalities

$$
\begin{gathered}
u=\xi+\Gamma_{l, \tau} l u, \\
\int_{a}^{b}\left[\int_{\tau}^{t}(l u)(\sigma) \mathrm{d} \sigma\right] \mathrm{d} t=0
\end{gathered}
$$

hold with

$$
\xi=u(\tau) .
$$

Conversely, if equations (4.1) and (4.2) hold with some $\xi \in X$, then $u$ is a solution of problem (1.4), (1.5) and, furthermore, equality (4.3) holds.

Prior to the proof of Lemma 11, we establish the following

Lemma 12. For an arbitrary $\xi$ from $X$, every solution of equation (4.1), if there are any, satisfies condition (1.5).

\footnotetext{
${ }^{1}$ Relation (3.7) should be understood similarly to (3.4) in Lemma 6; see Remark 7.
} 
Proof of Lemma 12. Let $u$ be a solution of (4.1) for some $\xi \in X$. Then, by virtue of (4.1) and Lemma 9, relation (4.3) holds. In view of (4.1) and (4.3), we have

$$
\begin{aligned}
\frac{1}{b-a} \int_{a}^{b} u(\sigma) \mathrm{d} \sigma-u(\tau) & \\
=\frac{1}{b-a} \int_{a}^{b}\left[\xi+\left(\Gamma_{l, \tau} l u\right)(\sigma)\right] \mathrm{d} \sigma-\xi-\left(\Gamma_{l, \tau} l u\right)(\tau) & =\frac{1}{b-a} \int_{a}^{b}\left(\Gamma_{l, \tau} l u\right)(\sigma) \mathrm{d} \sigma-\left(\Gamma_{l, \tau} l u\right)(\tau)
\end{aligned}
$$

whence, according to Lemma 9, it follows that

$$
\frac{1}{b-a} \int_{a}^{b} u(\sigma) \mathrm{d} \sigma-u(\tau)=\frac{1}{b-a} \int_{a}^{b}\left(\Gamma_{l, \tau} l u\right)(\sigma) \mathrm{d} \sigma .
$$

On the other hand, considering (3.3) and (3.2), we obtain the equality

$$
\int_{a}^{b}\left(\Gamma_{l, \tau} l u\right)(\sigma) \mathrm{d} \sigma=0
$$

which, combined with (4.4), proves our lemma.

Proof of Lemma 11. Let $u$ be a solution of system (4.1), (4.2). Then, according to Lemma 12, $u$ satisfies (1.5). Differentiation of equality (4.1), in view of (3.3), yields

$$
u^{\prime}(t)=(l u)(t)-l^{\square}(t) \Lambda_{l, \tau}^{-1} \int_{a}^{b}\left(\int_{\tau}^{s}(l u)(\sigma) \mathrm{d} \sigma\right) \mathrm{d} s, \quad t \in[a, b],
$$

whence we see that condition (4.2) guarantees the fulfillment of (1.4), i.e., $u$ satisfies both (1.4) and (1.5).

Conversely, if $u$ is a solution of problem (1.4), (1.5), then

$$
u(t)=\xi+\int_{\tau}^{t}(l u)(s) \mathrm{d} s, \quad t \in[a, b]
$$

with

$$
\xi:=u(\tau) .
$$

Therefore, by virtue of (4.5) and (1.5),

$$
\begin{aligned}
\int_{a}^{b}\left(\int_{\tau}^{s}(l u)(\sigma) \mathrm{d} \sigma\right) \mathrm{d} s & =\int_{a}^{b}[u(s)-\xi] \mathrm{d} s \\
& =\int_{a}^{b} u(s) \mathrm{d} s-(b-a) \xi \\
& =0 .
\end{aligned}
$$


Taking into account (3.3) and (4.6), we obtain

$$
\begin{aligned}
\left(\Gamma_{l, \tau} l u\right)(t)= & \int_{\tau}^{t}(l u)(s) \mathrm{d} s \\
& \quad-\int_{\tau}^{t} l^{\square}(\sigma) \mathrm{d} \sigma \Lambda_{l, \tau}^{-1} \int_{a}^{b}\left(\int_{\tau}^{s}(l u)(\sigma) \mathrm{d} \sigma\right) \mathrm{d} s \\
= & \int_{\tau}^{t}(l u)(s) \mathrm{d} s, \quad t \in[a, b],
\end{aligned}
$$

which means that (4.5) coincides with (4.1). Thus, we have shown that $u$ satisfies (4.1), (4.2).

\section{Iteration method for equation (4.1)}

The purpose of this section is to prove a statement (Lemma 13 below) is related to the iteration sequence ${ }^{2}$

$$
u_{k+1}:=\xi+\Gamma_{l, \tau} l u_{k}, \quad k=0,1, \ldots
$$

associated with equation (4.1). We suppose that Assumption 5 holds and, therefore, the expression in the right-hand side of (5.1) is well-defined.

Lemma 13. For an arbitrary $u_{0}$ from $C([a, b], X)$, sequence (5.1) can be represented alternatively as

$$
u_{k}=\xi+\left(\Gamma_{l, \tau} l\right)^{k} u_{0}, \quad k=1,2, \ldots
$$

Proof. It suffices to take into account Lemma 6 and apply Lemma 10 with $A:=$ $\Gamma_{l, \tau}$.

It follows from Lemma 13 that the structure of operator (3.3) guarantees the simplest possible dependence on the parameter $\xi$ in the iteration method corresponding to the parametrised equation (1.3).

Let us put

$$
\rho(l, \tau):=r\left(\Gamma_{l, \tau} l\right)
$$

and introduce the following

Assumption 14. $\rho(l, \tau)<1$.

Relation (5.3) makes sense because, as is easy to see from (3.3), the composition $\Gamma_{l, \tau} l$ is a continuous linear mapping from $C([a, b], X)$ to $C([a, b], X)$.

Lemma 15. Under Assumptions 1, 5, and 14, equation (4.1) possesses the unique solution

$$
u(t)=\xi, \quad t \in[a, b]
$$

for every $\xi$ from $X$.

\footnotetext{
${ }^{2} u_{0}$ in (5.1) is chosen in an arbitrary way
} 
Proof. By virtue of Lemma 13, the iteration sequence (5.1) corresponding to equation (4.1) admits representation (5.2). Assumption 14 guarantees that

$$
\lim _{k \rightarrow+\infty}\left(\Gamma_{l, \tau} l\right)^{k} u_{0}=0
$$

uniformly on $[a, b]$ for every $u_{0}$ from $C([a, b], X)$. In view of Lemma 13, this means that, uniformly on $[a, b]$,

$$
\lim _{k \rightarrow+\infty} u_{k}=0
$$

The uniqueness of solution (5.4) is obvious from Assumption 14. Indeed, if $w$ is another solution of equation (4.1) (with the same value of $\xi$ ), then the difference

$$
z(t):=\xi-w(t), \quad t \in[a, b]
$$

satisfies the equation

$$
z=\Gamma_{l, \tau} l z,
$$

which, by virtue of Assumption 14, has only the trivial solution.

\section{Unique solvability of problem (1.1), (1.2)}

We are now in a position to establish a theorem on the unique solvability of the mean value problems (1.4), (1.5) and (1.1), (1.2).

Proposition 16. Under 5, and 14, problem (1.4), (1.5) has only the trivial solution.

Proof. According to Lemma 11, every solution $u$ of problem (1.4), (1.5), if there are any, satisfies equation (4.1) for some $\xi \in X$. Lemma 15 guarantees the unique solvability of equation (4.1) for an arbitrary $\xi \in X$. Furthermore, the solution $u$ of (4.1) is represented by formula (5.4),

$$
u(t)=\xi, \quad t \in[a, b] .
$$

According to Lemma 11, function (5.4) is a solution of the mean value problem (1.4), (1.5) if, and only if

$$
\int_{a}^{b}\left[\int_{\tau}^{s}(l \xi)(\sigma) \mathrm{d} \sigma\right] \mathrm{d} s=0
$$

or, which is the same,

$$
\Lambda_{l, \tau} \xi=0 .
$$

By virtue of Assumption 5, relation (6.1) yields immediately $\xi=0$, whence, according to (4.1), it follows that $u=0$.

Proposition 17. Under Assumptions 1, 5, and 14, problem (1.1), (1.2) has a unique solution for arbitrary $q \in L([a, b], X)$ and $d \in X$. 
Proof. One can show that Assumption 1 guarantees the complete continuity of the mapping

$$
C([a, b], X) \ni u \longmapsto \int_{\tau}^{\cdot}(l u)(s) \mathrm{d} s .
$$

It is, therefore, easy to apply the Riesz-Schauder theory to conclude that (1.1), (1.2) is uniquely solvable for all $q$ and $\eta$ provided that the homogeneous mean value problem (1.4), (1.5) has only the trivial solution (see also [1]). Application of Proposition 16 completes the proof.

Remark 18. It is not difficult to show that Assumption 1 can be dropped in Proposition 17 , for which purpose one should establish slightly more general versions of Lemmata 11 and 13.

Remark 19. Proposition 17 is similar, in a sense, to Corollary 6.2 from [2, p. 162] (for the periodic problem) and Theorem 4.3.1 from [3, p. 146] (for a more general two-point problem), which concern linear systems of ordinary differential equations.

Remark 20. It can be shown that Assumption 14 is strict in the sense that it cannot be replaced by the inequality $\rho(l, \tau) \leq 1$.

\section{REFERENCES}

[1] Kiguradze, I. and PuŽA, B.: On boundary value problems for systems of linear functional differential equations, Czechoslovak Math. J., 47(2), (1997), 341-373.

[2] Kiguradze, I. T.: Initial and Boundary Value Problems for Systems of Ordinary Differential Equations. I. Linear Theory, Metsniereba, Tbilisi, 1997. (in Russian)

[3] Samoilenko, A. M., Laptinskit, V. N. and Kenzhebaev, K. K.: Constructive Methods of Investigating Periodic and Multipoint Boundary Value Problems, Inst. Math., Kiev, 1999. (in Russian) 